\title{
Determinants Of Perinatal Mortality In Bahir Dar Town Governmental Health Institutions, Northwest, Ethiopia, 2019: Prospective Unmatched Case Control Study
}

kebadnew mihretie ( $\sim$ kebadmulatu@gmail.com )

Bahir Dar University

DANIEL TAREKEGN WOREDE

Bahir Dar University

TAYE ABUHAY

Bahir Dar University

Research article

Keywords: perinatal mortality, determinants, Ethiopia

Posted Date: August 21st, 2019

DOl: https://doi.org/10.21203/rs.2.13314/v1

License: (c) (1) This work is licensed under a Creative Commons Attribution 4.0 International License.

Read Full License 


\section{Abstract}

Background: Perinatal mortality is defined as fetal death after 28 weeks of gestation and newborn death within seven days. Globally, more than 2.6 million stillbirths and over 2.7 million early neonatal deaths are estimated to occur each year. Each day an estimated 7,300 newborns die from complication during pregnancy, child birth and further neonatal causes and 7000 stillbirth, half of this occurs after labor had started. Almost all (98\%) takes place in developing countries and the magnitude of perinatal mortality in the study area was 44 per 1000 pregnancy. Objective: The objective of this study was to identify determinants of perinatal mortality in Bahirdar town governmental health institutions. Methods: Institutional based unmatched case control study was conducted .Cases were stillbirths and early neonatal deaths and controls were live births that were survived the first seven days after delivery. A total of 459 participants were involved in this study (153 cases and 306 controls) .Pretested, structured questioner with face to face interview was conducted and some data were also extracted using checklist from their medical records. Multivariable logistic regression analysis was done to analyze the data. A pvalue of $<0.05$ was considered as significant at $95 \%$ confidence interval and the strength of association was measured using odds ratio. Results: Antepartum hemorrhage (AOR 2.55,95\% Cl; 1.23-5.26), obstructed labour (AOR 3.11,95\% Cl; 2.00-8.38), prematurity (AOR 3.29,95\% Cl;1.86-5.81), first delay (AOR 2.61,95\% $\mathrm{Cl} ; 1.56-4.39$ ) and second delay (AOR 2.75,95\% Cl;1.49-5.11) were the determinants of perinatal mortality that increase risk of perinatal death. Whereas partograph use (AOR $0.24,95 \% \mathrm{Cl} ; 0.14-0.42$ ) and tertiary education (AOR $0.35,95 \% \mathrm{Cl} ; 0.17-0.71$ ) were found to be protective factors for perinatal mortality. Conclusion and Recommendation:The determinants of perinatal mortality were antepartum hemorrhage,prematurity, obstructed labour, first delay and second delay that were increase the risk of perinatal mortality whereas maternal tertiary education and partograph use during labour follow up were the protective factors. But the risk factors were easily identifiable and manageable with the existing health care services while health partograph use in labour follow up and educating females to tertiary education level is better, first and second delay need to avoided during

\section{Introduction}

Perinatal mortality is defined as a fetal death (stillbirth) and early neonatal death. Stillbirth is a baby born with no sign of life, weighing more than 1000 gram or with more than 28 completed weeks of gestation and early neonatal death is a death in the first week of life after delivery(1).

Due to disparity in the quality of services provided for pregnant women and their babies' perinatal mortality in developing nation is about five times higher than developed nations(2).

Currently, the quality of antenatal and perinatal care provided to the community is best judged by the perinatal mortality rate. Annually 2.6 million stillbirths and 2.7 million early neonatal deaths occurs worldwide(3).In the year 2015, each day an estimated 7,300 newborns die from complication during pregnancy, child birth and further neonatal causes worldwide and 7000 still births. From those $98 \%$ of deaths occurs in developing countries(4). 
Although, there was remarkable decline (40\%) in perinatal mortality in Ethiopia in the last two decades, it is still an important public health problem about 66 to 124 deaths per 1000 births in health institution and 25 to 52 deaths per 1000 births in the community $(5,6)$. The community based study conducted in Dabat, Ethiopia showed that perinatal mortality rate was 50.22 deaths per 1000 births(7). Ethiopian 2016 EDHS finding showed that perinatal mortality rate was 44 deaths per 1000 pregnancies in Amhara region(8).

Perinatal mortality as health indicator plays an important role in providing the information needed to improve the health status of the pregnant women and their newborn.

Identifying determinants of perinatal mortality is very important for decision makers in building and implement strategies to improve the care provided to the pregnant mothers and their newborns.

Most studies identified that hemorrhagic disorders, hypertensive disorders, obstructed labor, prolonged labour, neonatal sepsis, asphyxia, congenital anomalies, prematurity, advanced maternal age were the risk factors and ANC follow up, TT vaccination, use of Partograph during labor, doctor birth attendant were protective factors associated with perinatal mortality $(5,9-14)$. But first delay, second delay (delay in reaching care) and maternal behavioral factors such as alcohol use, smoking,drug use during pregnancy, nutritional status, chat chewing were not documented and understood in the study area. Therefore, this institutional based unmatched case control study design was conducted to identify the potential determinants of perinatal mortality in Bahir-dar town governmental health institutions using incident cases.

\section{Methods And Materials}

Institutional based unmatched case control study was conducted in Bahir-dar town governmental health institutions from $1^{\text {st }}$ March to $30^{\text {th }}$ May; 2019.

The source population was all cases of still birth, early neonatal deaths with in the first week of life and live births at least 1000 gram birth weight or approximately 28 or above weeks of gestation in the study period. Those stillbirths and early neonatal deaths after 28 weeks of pregnancy for cases and those live births after 28 weeks of gestation were eligible for study whereas sill births, early neonatal deaths and live births with maternal mortality were excluded.

The sample size was determined based on determinant factors associated with perinatal mortality in the previous study(6).Using epi -info version 7 , with the assumption of $80 \%$ power, $1: 2$ case to control ratio, $95 \%$ confidence interval and $5 \%$ non-response. A total of 459 (153 cases and 306 controls) participants were included in the study.

The sample was proportionally drawn based on average delivery rate six months prior to data collection from seven health institutions in the town. Data were collected in the maternity wards, neonatal intensive care units and postnatal clinics, all mothers whose babies died in the health institutions during the perinatal period from $1^{\text {st }}$ March to $30^{\text {th }}$ May, 2019 were recruited as cases consecutively until the required 
sample size was reached. And two controls per case were selected using systematic sampling technique from mothers who had given birth in the study period using delivery log book. Perinatal mortality were outcome variable $(1=$ died and $0=$ alive $)$ whereas:-maternal age, maternal occupation, maternal educational status, maternal marital status, prematurity, mode of delivery, fetal presenting part, birth interval,ANC visit, timing of ANC initiation,TT vaccination, preceding family planning use, Iron-folate intake during pregnancy, history of perinatal death, history of abortion, hemorrhagic disorder, obstructed labour, prolonged, labour hypertensive disorder, HIV, DM, anemia, STI, HBV infection, birth weight,Congenital anomaly, neonatal jaundice, neonatal sepsis, bleeding, accidental injury/fall,partograph use, first delay, second delay, qualification of birth attendant, smoking, chewing khat, drug use, alcohol consumption were independent variables

Case: is defined as newborn at least 1000 gram of birth weight or corresponding to 28 and above weeks of gestation in the health institutions either as a still birth or born alive but died within seven days after delivery. Gestational age was determined using last normal menstrual period (LNMP) or ultrasound report. Amenorrhea of 9 months was approximated to 40 weeks of gestation

Control: is defined as live births at least 1,000 gram of birth weight or corresponding to approximate 28 weeks of gestation in health institutions of the study area and survived the first seven days after delivery. Delay in seeking labour care (first delay): is refers to the time spent at home before decision is made to seek labour care (it was considered delayed if the mother stay at home $\geq 3$ hours after labour had started).Delay in reaching care (second delay).is the problem related to reach the health institutions after decision had made to do there(it was considered delayed if the mothers were faced transport/ambulance problem during transportation either from home to health institutions or from health institutions to health institutions during referral on labour (it was measured as yes/no)..Prematurity: is defined as a birth that takes place before 37 weeks of gestational (it was measured as yes if less than 37 weeks of gestational age and $n o$ if $\geq 37$ weeks of gestation).

Data were collected by 7 midwife and neonatal nurses who were worked in the health institutions by face to face interview using Amharic version of the questioner from all eligible mothers and some data were also collected from medical records of mothers and foetus/new-borns, admission history, referral sheets, delivery follow up sheets, delivery summery, neonatology and delivery log books, death summery and death confirmation sheets using checklist.

A questioner was translated to Amharic, the working language in the study area by two person one medical professional and the other English language professional, translated back into English to keep consistency. Two days training was given for data collectors and supervisors. The overall activity was supervised regularly by two supervisors who are MSC holder midwife and experienced in this type of activity. Pretest was done before the actual data collection.

Data were coded, entered and cleaned using epi-info version 7 then analysis was performed using SPSS version 23 software after imported from epi-info. Frequency distribution was used to describe the characteristics of the study population. Bi-variable and multivariable logistic regression analysis was 
done. Those variables associated at bi-variable analysis with $\mathrm{p}$-value $<0.25$ were entered into multiple logistic regression analysis. Multiple logistic regression model was fitted using backward elimination technique. The fitness of model was checked using Hosmer-Lemshow test with $p$-value was $>0.05$. Odds ratio with $95 \%$ confidence interval was used to measure strength of association, those variables whose $p$ value less than 0.05 were taken as significant.

\section{Results}

A total of 442 (148 cases and 294 controls) were interviewed with response rate of $96.3 \%$ (96.5\% for cases and $96.1 \%$ for controls). The mean age of the case and control mothers were $28.22 \pm 7.50$ (SD) and $26.23 \pm 4.88$ standard deviation(SD) years respectively. About 264(89.8\%) of control mothers and 114 $(77.0 \%)$ of case mothers were found in the age category of $20-34$ years. About $89(30.3 \%)$ and $21(14.2 \%)$ of control and case mothers had attended tertiary education level respectively. With regard to current occupational status, only $73(24.8 \%)$ of control mothers employed when compared to case mothers $42(28.4 \%)$ and housewife accounted $102(34.7 \%)$ in control mothers and $41(27.7 \%)$ in case mothers. Majority of control mother, $248(84.4 \%)$ and $114(77.0 \%)$ case mothers were married. About $209(71.1 \%)$ mothers with livebirths were lived in urban areas as compared with $70(47.3 \%)$ case mothers.

Of all $107(36.4 \%)$ and $57(38.5 \%)$ of control and case mothers were delivered at the first time (primgravida) respectively. Whereas, $156(53.1 \%)$ of controls and $69(46.6 \%)$ of case mothers were multigravida. About, 249 (84.7\%) of control and 127 (85.8\%) of case mothers had antenatal care visit for the current pregnancy respectively. From those mothers who had antenatal care visit for current pregnancy, 205(69.7\%) and 96(64.9\%) were started antenatal care follow up within three months (first trimester) respectively for control and case mothers.

With regard to tetanus toxoid vaccination status, about, $244(83.0 \%)$ control mothers and $96(64.9 \%)$ case mothers had received tetanus toxoid vaccine, and 194(66.0\%) controls mothers and 70(47.3\%) case mothers had taken iron-folate pill during current pregnancy. A total of $232(78.9 \%)$ of control and $86(58.1 \%)$ of case mothers were used family planning before the current pregnancy. Eight $(2.7 \%)$ of control and $9(6.1 \%)$ of case mothers had previous history of perinatal death. Regarding to mode of delivery, $206(70.1 \%)$ of control mothers and $90(60.8 \%)$ of case mothers had delivered with spontaneous vaginally, while, the remaining $64(21.8 \%)$ of control and $44(29.7 \%)$ of case mothers had given by assisted vaginal delivery, and $24(8.2 \%)$ of control and $14(9.5 \%)$ of case mothers delivered with caesarean sections.

From maternal medical complications, antepartum hemorrhage was accounted $25(8.5 \%)$ in control and $36(24.3 \%)$ case mothers, anemia $8(2.7 \%)$ among control mothers and $13(8.8 \%)$ in case mothers, hypertensive disorders $15(5.1 \%)$ and $23(15.5 \%)$ from control and case mothers respectively, whereas from obstetric complication, obstructed labour was accounted in $17(5.8 \%)$ and $46(31.1 \%)$ from control and case mothers respectively. 
From all participants in the study, $167(56.8 \%)$ of control mothers and $86(58.1 \%)$ of case mothers had delivered female newborns of those, $14(4.8 \%)$ of control mothers and $9(6.1 \%)$ of case mothers delivered twin births. About $41(13.9 \%)$ of Control mothers and $40(27.0 \%)$ of case mothers had delivered low birth weight ( $<2500 \mathrm{gm})$ newborns, while the remaining, $253(86.1 \%)$ and $108(73.0 \%)$ of control and case mothers had delivered normal birth weight newborns.

With regard to neonatal complications, about, $21(7.1 \%)$ of control and $12(8.1 \%)$ of cases accounts neonatal sepsis, $16(5.4 \%)$ of controls and $13(8.8 \%)$ of cases, $9(3.1 \%)$ controls and $11(7.4 \%)$ of cases and $40(13.6 \%)$ of controls and $72(48.6$. \%) of cases had congenital anomaly, neonatal jaundice and prematurity respectively.

\section{The determinants of perinatal mortality}

Binary logistic regression analysis was done to identify the independent determinants of perinatal mortality. Both bi-variable and multivariable logistic regression analysis revealed that literacy, antepartum hemorrhage, prematurity, obstructed labour, delay in seeking labour care, transport problem and partograph use were the determinants of perinatal mortality( Table:1)..

Table 1: The determinants of perinatal mortality in Bahir-dar town governmental health institutions, northwest, Ethiopia, 2019 
Variable

Perinatal outcome COR(95\%CI)

AOR (95\%)

Controls Cases

Maternal education

No education

55(18.7) 59(39.9)

1

1

Primary

75(25.5) 32(21.6) 0.39(0.23-0.69)

$0.51(0.25-1.011)^{*}$

Secondary

75(25.5) 36(24.3)0.45(0.26-0.77)

$0.55(0.27-1.11) *$

Tertiary

89(30.3) 21(14.2)0.22(0.12-0.40)

0.35(0.17-0.71)

Prematurity

No 254(86.4) 76(51.4)

1

1

Yes 40(13.6) 72(48.6)

6.01(3.78-9.57)

$3.29(1.86-5.81)$

)bstructed labour

\begin{tabular}{|c|c|c|c|}
\hline No $277(94.2)$ & 102(68.9) & 1 & 1 \\
\hline Yes 17(5.8) & $46(31.1)$ & 7.34(4.03-13.40) & $3.11(2.00-8.38)$ \\
\hline
\end{tabular}

intepartum hemorrhage No 269(91.5) 112(75.7)

1

1

Yes 25(8.5) 36(24.3) 3.45(1.98-6.03) 2.55(1.23-5.26)

ransport problem

No 261(88.8) 85(57.4)

1

1

Yes 33(11.2) 63(42.6)

$5.86(3.60-9.54)$

$2.75(1.49-5.11)$

)elay in seeking

No 177(60.2) 48(32.4)

1

1

abour care

Yes117(39.8) 100(67.6)

$3.15(2.08-4.78)$

$2.61(1.56-4.39)$

'artograph use

No 49(16.7) 66(44.6)

1

1

Yes245(83.3) 82(55.4)

$0.24(0.16-0.39)$

$0.24(0.14-0.42)$

$1=$ reference category * non-significant

\section{Discussion}

The odds of experiencing perinatal mortality were decreased by $65 \%$ among mothers who had attended tertiary education compared to those who had no education. This study was in line with studies conducted in Dabat, Amhara region(7), Addis Ababa(9) and Kigali, Rwanda(10).

This might be due to literacy improve economic status, access to health care and improves birth spacing.

The odds of experiencing perinatal mortality were 2.55 times more likely among mothers who had antepartum hemorrhage during pregnancy than those who had no APH (AOR 2.55, 95\% Cl; 1.23-5.26). The finding of this study was consistent with the studies conducted in Hawassa, Ethiopia(11),Kenya(12), 
Zimbabwe(13), Rural Bangladesh (14) and Nepal(15). This might be due to placental abnormalities that could results in perinatal deaths. But a case control study conducted in northern Tigray, Ethiopia (16), finding indicated that antepartum hemorrhage was not associated with perinatal mortality.The result of the study was disagree with this study. The reason might be due to differences in quality of health care service availability and accessibilities that could help to early detection and management of antepartum hemorrhage during pregnancy.

Prematurity was one of the strongest determinants of perinatal mortality in this study. The odds of experiencing perinatal mortality were 3.29 fold more likely among premature births compared to those mature births (AOR 3.29, 95\% Cl;1.86-5.81).This finding was in line with the studies conducted in Gojjam(6),Tigray(16), Oromia(17),Tanzania(18),Zimbabwe(13),Ghana(19) and Kerala, India(20).The study also conducted in Haryana, India (21) showed that odds of perinatal mortality in premature deliveries were about 3.5 times more likely when compared to term deliveries. The other case control study conducted in Brazil(22) also indicated that prematurity was associated with perinatal mortality. The reason might be due to vulnerability of premature births to many complications, including respiratory distress syndrome, compromised immune system, cardiovascular disorders, and infections.But the study conducted in Jimma specialized hospital, Ethiopia(23) showed that preterm births were not associated with perinatal mortality. The result of the study was disagreed with the current study. The reason might be due to difference in nutritional status of the mothers that gave births.

Obstructed labour was the other determinant factor associated with perinatal mortality. The result of this study showed that 3.11 fold higher odds of perinatal mortality were observed on women who had obstructed labour than those who had no obstructed labour (AOR 3.11,95\% Cl;2.00-8.38). The result of this study was consistent with the study conducted in Hawassa(11),Northern Ghana(24) and Tanzania(25).This might be due to perinatal asphyxia, that those mothers who had obstructed labour might have the greatest perinatal asphyxia.

Partograph use during labour follow up was also found to be protective factor for perinatal mortality. The odds of experiencing perinatal mortality were $76 \%$ less likely among mothers whose labour was monitored using partograph compared to those mothers whose labour was not monitored using partograph (AOR 0.24, 95\% Cl; 0.14-0.42). Unmatched case control study conducted in Addis Ababa among public health deliveries indicated that the odds of experiencing perinatal mortality were decreased by $65 \%$ compared to women who had no labour follow up using Partograph (9). The other case control study conducted in Northern Tigray(16) revealed that the odds of perinatal mortality were $90 \%$ less likely among women whose labour was monitored using partograph compared to those mothers whose labour was not monitored using partograph. The finding was agreed with this study. This might be due to using partograph may be helpful in early detection of fetal and maternal complications. Partograph is recommended by world health organization and its appropriate use is can help to detect abnormalities of fetus during labour and delivery. 
Delay in seeking labour care was the other determinants of perinatal mortality in the current study. The odds of experiencing perinatal mortality were 2.61 times more likely among mothers who were delay in seeking labour care compared to those mothers who were not delay (AOR 2.61, 95\% Cl; 1.56-4.39). This finding was agreed with the study conducted in northern Tigray (16).This might be due to failure to recognize signs of complications, failure to perceive the severity of illness, cost considerations, previous negative experiences with the healthcare system, and transportation difficulties.

Perinatal mortality was significantly associated with transport problem. The odds of perinatal mortality were 2.75 times more likely among mothers who had encountered transport (ambulance) problem compared to those mothers who had no transport problem during labour (AOR 2.75, 95\% Cl; 1.495.11).The result of this study was consistent with a study conducted in Uganda(26). This might be due to distance from a mother's home to health institutions or from institutions to institutions during referrals, the condition of roads, and a lack of emergency transportation. The time taken to reach health facility has been indicated as risk factor for perinatal deaths in developing nations(2). A large number of mothers had to travel more than an hour to reach the referral hospitals and other health facilities and most of the public transportations are difficult for mothers who are on labour the best option for this is ambulance serves as government tries to access it to all health centers and hospitals. The risk of recall bias by mothers judged to be low because, they were interviewed shortly after delivery or newborn death was the strength of the study.

\section{Limitations Of The Study}

The health institution based study might have an over-representation of the determinants of perinatal mortality, as more complicated cases are referred and since the data was collected in institution only the perinatal deaths in the community after discharge were not considered.

\section{Conclusion And Recommendation}

The determinants of perinatal mortality in the study area was largely due to medical, obstetric, neonatal complications that are easily identifiable and manageable with the existing basic obstetric and neonatal care whereas health care and socio-demographic factors were also the important determinants that were associated in this study that needs further encouragement in partograph use in labour follow up and educating females to tertiary education. Health care providers had to be early detect and manage antepartum hemorrhage, obstructed labour and prematurity and partograph use, transports services (ambulance), and female tertiary education need to be encouraged and Counseling and health education about delay in seeking labour care is needed finally identifying the reason of first delay needs further research and qualitative research needs to be done on $3^{\text {rd }}$ delay at the health institutions.

\section{Declarations}

Ethical approval 
Ethical approval was obtained from Bahir-dar University, collage of health science, school of public health Institutional review board. Official letter of co-operation was written to selected health institutions and permission was obtained from respective health institutions concerned officials. All eligible parents who were at the time of the event were given detailed information about the study in local language by the data collectors and then written informed consent was taken from every mother who voluntarily agreed to participate in the study. The right of the respondent was informed and respected to withdraw or not to participate in the interview. Confidentiality of the information collected was maintained.

\section{Authors' Contributions}

DT, KM, and TA conceived the study, involved in the study design, data analysis, drafted the manuscript and critically reviewed the manuscript. All authors read and approved the final manuscript

\section{Acknowledgments}

we would like to express our deepest gratitude to BDU, data collectors, participants of the study and health professionals in each health institutions who supported us.

\section{Funding}

The study was funded by Bahir-dar University

\section{References}

1.Organization WH. The WHO application of ICD-10 to deaths during the perinatal period: ICD-PM. WHO website 2016:77.

2.Yadufashije C, Sangano GB, Samuel R. Factors Associated with Perinatal Mortality in Africa. SSRN Electronic Journal. 2017.

3.Blencowe H, Cousens S, Jassir FB, Say L, Chou D, Mathers C, et al. National, regional, and worldwide estimates of stillbirth rates in 2015, with trends from 2000: a systematic analysis. The Lancet Global Health. 2016;4(2):e98-e108.

4.World Health Organization U. Making Every Baby Count: Audit and Review of Stillbirths and Neonatal Deaths. 2016.

5.Berhan Y, Berhan A. Perinatal mortality trends in Ethiopia. Ethiopian journal of health sciences. 2014;24 Suppl(0 Suppl):29-40.

6.Yirgu R MM, Sibley L, Gebremariam. (2016) Perinatal Mortality Magnitude, Determinants and Causes inWest Gojam: Population-BasedNested Case-Control Study. PLoS ONE:. 11(7)(e0159390.). 
7.Andargie G, Berhane Y, Worku A, Kebede Y. Predictors of perinatal mortality in rural population of Northwest Ethiopia: a prospective longitudinal study. BMC Public Health. 2013;13:168.

8.Central Statistical Agency - CSA/Ethiopia, ICF. Ethiopia Demographic and Health Sruvey 2016. Addis Ababa, Ethiopia: CSA and ICF; 2017.

9.Getiye Y, Fantahun M. Factors associated with perinatal mortality among public health deliveries in Addis Ababa, Ethiopia, an unmatched case control study. BMC Pregnancy Childbirth. 2017;17(1):245.

10.Musafili A, Essen B, Baribwira C, Selling KE, Persson LA. Social equity in perinatal survival: a casecontrol study at hospitals in Kigali, Rwanda. Acta Paediatr. 2015;104(12):1233-40.

11.Bayou G, Berhan Y. Perinatal mortality and associated risk factors: a case control study. Ethiopian journal of health sciences. 2012;22(3):153-62.

12.Yego F, D'Este C, Byles J, Nyongesa P, Williams JS. A case-control study of risk factors for fetal and early neonatal deaths in a tertiary hospital in Kenya. BMC Pregnancy Childbirth. 2014;14(389):389.

13.Tachiweyika E, Gombe N, Shambira G, Chadambuka A, Mufuta T, Zizhou S. Determinants of perinatal mortality in Marondera district, Mashonaland East Province of Zimbabwe, 2009: a case control study. The Pan African medical journal. 2011;8:7-.

14.Owais A, Faruque AS, Das SK, Ahmed S, Rahman S, Stein AD. Maternal and antenatal risk factors for stillbirths and neonatal mortality in rural Bangladesh: a case-control study. PLoS One. 2013;8(11):e80164.

15.K CA, Nelin V, Wrammert J, Ewald U, Vitrakoti R, Baral GN, et al. Risk factors for antepartum stillbirth: a case-control study in Nepal. BMC Pregnancy Childbirth. 2015;15:146.

16.Goba GK, Tsegay H, Gebregergs GB, Mitiku M, Kim KA, Alemayehu M. A facility-based study of factors associated with perinatal mortality in Tigray, northern Ethiopia. Int J Gynaecol Obstet. 2018;141(1):1139 .

17.Roro EM, Sisay MM, Sibley LM. Determinants of perinatal mortality among cohorts of pregnant women in three districts of North Showa zone, Oromia Region, Ethiopia: Community based nested case control study. BMC Public Health. 2018;18(1):888.

18.Macheku GS PR, Oneko O, Mlay PS, Masenga G, Obure J,, MJ. M. Frequency, risk factors and fetomaternal outcomes of abruptio placentae in Northern Tanzania: a registry-based retrospective cohort study. BMC Pregnancy Childbirth. 2015;15(242).

19.A Kraft C. Identifying Risk Factors Associated With Intrapartum Stillbirth and Neonatal Mortality in Rural Ghana. Neonatology \& Clinical Pediatrics. 2015;2(2):1-3. 
20.Brahmanandan M, Murukesan L, Nambisan B, Salmabeevi S. Risk factors for perinatal mortality: a case control study from Thiruvananthapuram, Kerala, India. International Journal of Reproduction, Contraception, Obstetrics and Gynecology. 2017;6(6):2452.

21.Neogi SB, Negandhi P, Chopra S, Das AM, Zodpey S, Gupta RK, et al. Risk Factors for Stillbirth: Findings from a Population-Based Case-Control Study, Haryana, India. Paediatr Perinat Epidemiol. 2016;30(1):56-66.

22.Moura PMSS, Maestá I, Rugolo LMSS, Angulski LFRB, Caldeira AP, Peraçoli JC, et al. Risk factors for perinatal death in two different levels of care: a case-control study. Reprod Health. 2014;11(1):11-.

23.Aragaw YA. Perinatal Mortality and associated Factor in Jimma University Specialized Hospital, South West Ethiopia. Gynecology \& Obstetrics. 2016;6(11).

24.Engmann C, Walega P, Aborigo RA, Adongo P, Moyer CA, Lavasani L, et al. Stillbirths and early neonatal mortality in rural Northern Ghana. Trop Med Int Health. 2012;17(3):272-82.

25.Rose Mpembeni NM JR, Mughamba J (2014). Perinatal Mortality and Associated Factors Among Deliveries in Three Municipal Hospitals of Dar Es Salaam, Tanzania. J Pediatr Neonatal Care 1(4): (00022.).

26.Beatrice Akello 1 EN, Christine Zirabamuzaale 2 and Christopher Garimoi Orach 2. risk factors for perinatal mortality in Arua regional referral hospital, west nile,Uganda. East African Journal of Public Health. 2008;5(3). 